\title{
Water Suspension Infiltration with Adsorption Including Heat Transport in Unsaturated-Saturated Porous Media
}

\author{
Jozef Kačur ${ }^{1,2}$, Patrik Mihala ${ }^{2,1}$ \\ ${ }^{1}$ Faculty of Civil Engineering of the Slovak University of Technology \\ Radlinského 11, 81005 Bratislava, Slovakia \\ Jozef.Kacur@fmph.uniba.sk; pmihala@gmail.com \\ ${ }^{2}$ Faculty of Mathematics, Physics and Informatics, Comenius University in Bratislava \\ Mlynská dolina, 84248 Bratislava, Slovakia
}

\begin{abstract}
Transportation of heat and water suspension (water and silt) in unsaturated-saturated porous media is considered. Moreover, the water in suspension is contaminated and this contaminant is adsorbed by the porous media matrix. Also, the inner heat exchange between water suspension and the matrix of porous media is considered in the complex model. The deposition of silt in the matrix is characterized by a filtration function and the contaminant adsorption is modeled by a sorption isotherm. The mathematical model includes a coupled system for the water suspension infiltration, silt, and contaminant (uniformly mixed in the water) transport with dispersion and their deposition and adsorption in porous media. Filtration function expresses the rate of silt deposition depending on the amount of (immobile) deposited silt. Contaminant adsorption is modeled in terms of the contaminant concentration in suspension, the amount of adsorbed contaminant, and the rate of adsorption. The main goal is to develop a suitable numerical approximation that can be applied to the solution of direct and inverse problems. In the numerical experiments, we demonstrate the correctness and the effectiveness of the used method.
\end{abstract}

Keywords: suspension and heat transport, heat energy exchange, silt deposition, contaminant adsorption, porous media, numerical modelling

\section{Introduction}

The flow of water suspension (silt particles mixed in water) in porous media was modeled and intensively discussed in the last decade. Silt particles are retained at the pores, some of them become immobile and some of them continue transportation. Kinetics of particle retention depends on the concentration of particles in suspension, on the amount of already retained immobile particles and the speed of water suspension. The retention process is much more complex, influenced also by the ionic strength of microparticles and physicochemical mechanisms including contact with porous media. In fact, also repulsive interaction occurs, and thus both phenomena, deposition, and release of particles, participate. For practical applications, not all attributes and influences could be included in the model. The deposition of particles based on the mechanical mechanism modeled in terms of filtration function was presented by Herzig et al [13]. This model was simple, expressing just conservation of the mass of deposited particles and particles in suspension. There, the kinetics of particle retention is dependent on their concentration in water suspension and level of retained ones expressed in terms of the filtration function. Determination of filtration function (dependent on the amount of retained particles) was discussed in a series of papers, see [11,14], and citations there. In spite of elegant analysis the used method requires very special (smooth and obeying some structural conditions) input data which represent inflow/outflow measurements of concentration. A more complex model has been discussed in [12] (see also citations there) where transport of silt solute together with particle transport was considered containing deposition/release phenomenon. The kinetics of deposition/release is expressed in terms of silt concentration and water velocity changes. Suspension flow is assumed in fully saturated porous media. The flow speed in [13] is constant and in [12] is assumed in an analytical form. Besides the filtration property of porous media also the distribution of particle deposition in porous media is of interest.

In our contribution, we discuss a more complex 3D model for suspension flow where we allow also partial saturation. Moreover, we take into account the heat energy transport with its transmission between suspension and the porous media. The contaminant in suspension is transported and adsorbed by the matrix. Both deposited silt and adsorbed contaminant 
change porosity and hydraulic permeability. Also the temperature of suspension changes the viscosity and consequently the hydraulic permeability. The suspension flow in unsaturated porous media is governed by gravitation and capillarity phenomenon which depends on suspension saturation in porous media. Thus the complex system is strongly coupled. Mathematical models for the flow of water and contaminant transport are discussed in monographs, e.g. $[1,9,10]$ and numerical modeling with inverse problems we have realized in [6] for 1D case.

The governing equations of our system consist of a strongly nonlinear and degenerate elliptic-parabolic system. The suspension flow is governed by Richard's type equation where capillary pressure and hydraulic permeability versus suspension saturation is assumed in an empirical model by van Genuchten/Mualem [4]. Moreover, these fundamental flow relations and porosity depend on deposited silt, adsorbed contaminant, and suspension viscosity which depends on suspension temperature.

The heat exchange with the porous media matrix we have analyzed in our previous contribution [15] where only clean water has been considered. In this contribution, we will focus on the heat exchange of suspension with the porous media matrix and the filtration only in the deposition mode. Our main goal is the development of a correct and efficient numerical method for solving the direct (when all model parameters are available) and inverse problem in which we determine the heat transmission coefficient and the filtration function. There, the inflow/outflow measurements in laboratory experiments are used. In the solution of inverse problems, we consider the 3D sample in cylindrical form. The 3D sample enables us to use a large scale of experimental scenarios choosing suitable boundary conditions. We choose optimal of them with respect to the reliability of determined model parameters and the technical simplicity of realized additional measurements needed for the inverse method. The experiment is schematically drawn in Fig. 1.

In Section 2 we present the mathematical model and in Section 3 its numerical approximation. In Section 4 we discuss the solution of the inverse problem in which filtration function will be determined. Numerical experiments are introduced in Section 5, where the solution of direct and inverse problems are presented.

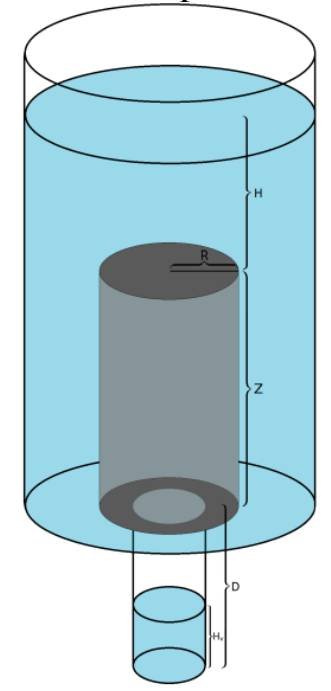

Fig. 1: Setting of the sample in experimental scenarios.

Our numerical method is based on operator splitting and finite volume method with flexible time discretization where successively along with small time interval we separately solve suspension flow, transport of silt and contaminant with deposition and adsorption, heat transport by suspension and then in the matrix including the heat exchange. In the approximation of the suspension flow model, we follow the approximation strategy introduced in [3] and also used in well-known software Hydrus (see [2]).

\section{Mathematical model}




\subsection{Suspension flow model}

The flow is modelled by the hydraulic permeability $K=K_{s} k(h)$, with

$$
K_{s}=\kappa_{0} \frac{\rho g}{\mu}
$$

where $\rho$ and $\mu$ are the density and the dynamical viscosity of the suspension, respectively. The coefficient $\kappa_{0}$ depends only on the structure of the porous medium and $g$ is the gravitational acceleration. Denote by $\theta$ suspension saturation, $\theta f$ amount of silt in suspension and by $h$ the pressure head of suspension.

The model function $k(h)$ is linked with the capillary forces dependent on the corresponding effective saturation $\bar{\theta}$. (see van Genuchten [4]). We note that these parameters depend on suspension temperature $T_{w}$, deposited material $\mathrm{S}=\left(S_{1}, S_{2}\right)$, where $S_{1}$ is adsorbed contaminant and $S_{2}$ is deposited silt. By $C_{w}$ we denote contaminant concentration in suspension. Flux of water suspension $\overrightarrow{q_{0}}$ is the sum of water and silt fluxes $q_{w}, q_{s}$ and is driven by gravitation and capillary pressure, expressed in the terms of hydraulic permeability and saturation using van Genuchten-Mualem empirical model.

We consider $K$ in more general form

$$
K\left(T w, C_{w}, f, S, h\right)=\frac{\theta_{s}-\left(S_{1}+S_{2}\right)}{\theta_{s}} K_{s}\left(T_{w}, C_{w}, f\right) k(h)
$$

since the deposited material decreases the original porosity $\theta_{s}$.

Here, $K_{S}=K\left(T_{w}, C_{w}, f, S, 0\right)$ is the hydraulic permeability in fully saturated porous media. We consider $k$ in the van Genuchten/Mualem empirical form (see [4])

$$
k(\bar{\theta})=\bar{\theta}^{\frac{1}{2}}\left(1-\left(1-\bar{\theta}^{\frac{1}{m}}\right)^{m}\right)^{2}
$$

where

$$
\bar{\theta}=\frac{\theta}{\theta_{s}-\left(S_{1}+S_{2}\right)}
$$

with (originally) fully saturated $\theta_{s}$ and residual $\theta_{r}$ water contents, respectively. The capillary pressure vs. saturation (fundamental relation) we consider in the form

$$
\bar{\theta}=\frac{1}{\left(1+(\alpha h)^{n}\right)^{m}}
$$

where $n>1, m=1-\frac{1}{n}$ and $\alpha<0$ are the soil parameters in the van Genuchten-Mualem (empirical) model (see [1,4]).

In the saturated zone we have (Darcy's law) $k(h) \equiv 1$. The influence of dynamical viscosity on $C_{w}, f, T_{w}$ can be found on tables for discrete values of variables and we use a spline interpolation of them in our computations. Richard's type equation modelling the contaminated suspension flow with heat transport reads as follows

$$
\begin{gathered}
\partial_{t} \theta+\nabla\left(q_{w}+q_{s}\right)+\partial_{t}\left(S_{1}+S_{2}\right)=0 \\
\partial_{t}\left(\theta C_{w}\right)+\operatorname{div}\left(\overrightarrow{q_{0}} C_{w} \theta-\theta \mathrm{D} \nabla C_{w}\right)+\partial_{t} S_{1}=0 \\
\partial_{t} S_{1}=\kappa\left(\Psi_{1}\left(C_{w}\right)-S_{1}\right) \\
\partial_{t}(\theta f)+\nabla q_{s}+\partial_{t} S_{2}=0 \\
\partial_{t} S_{2}=\Psi_{2}\left(S_{2}\right) \theta f \\
c_{v} \partial_{t}\left(\theta T_{w}\right)-\operatorname{div}\left(-c_{v} \vec{q} T_{w}+\theta \mathrm{D} \nabla T_{w}\right)=\sigma \theta\left(T_{m}-T_{w}\right) \\
c_{m} \partial_{t} T_{m}-\lambda \Delta T_{m}=\sigma \theta\left(T_{w}-T_{m}\right) .
\end{gathered}
$$


The suspension flux is

$$
\overrightarrow{q_{0}}(x, t)=-K\left(\bar{\theta}, C_{w}, f, S\right)\left(\nabla h-\left(1-f+f \frac{\rho_{s}}{\rho_{w}}\right)\right)
$$

then the water and silt flux is

$$
q_{w}=(1-f) \overrightarrow{q_{0}}, \quad q_{s}=f \overrightarrow{q_{0}}
$$

\subsection{Contaminant and silt transport model}

The flux of dissolved contaminant with concentration $C_{w}$ denoted by $\mathbf{J}_{C_{w}}$ is

$$
\mathbf{J}_{C_{w}}=\theta\left(\overrightarrow{q_{0}} C_{w}-\mathrm{D} \nabla C_{w}\right)
$$

Here, $\mathbf{v}$ is the seepage velocity of the contaminated suspension linked with the flux $\overrightarrow{q_{0}}$.

Denote by $\mathrm{D}$ the dispersion matrix with the components

$$
D_{i j}=\left(D_{0}+\alpha_{T}|\mathrm{v}|\right) \delta_{i j}+\frac{v_{i} v_{j}}{|\mathrm{v}|}\left(\alpha_{L}-\alpha_{T}\right),
$$

where $\alpha_{L}, \alpha_{T}$ are longitudinal and transversal dispersive coefficients, respectively, $\delta_{i j}$ is the Kronecker delta and $D_{0}$ is the molecular diffusion coefficient. Then, the contaminant transport model is

$$
\partial_{t}\left(\theta C_{w}\right)+\operatorname{div}\left(\overrightarrow{q_{0}} C_{w} \theta-\theta \mathrm{D} \nabla C_{w}\right)=-\rho_{m} \partial_{t} S_{1}
$$

where $\rho_{m}$ is the density of the porous media matrix and $S_{1}$ is adsorbed contaminant by the unit mass of porous media matrix. The adsorption of the contaminant is governed by the ordinary differential equation (see [1])

$$
\partial_{t} S_{1}=\kappa\left(\Psi_{1}\left(C_{w}\right)-S_{1}\right),
$$

where $\kappa$ is the sorption rate coefficient describing adsorption kinetics and $\Psi_{1}$ is a sorption isotherm, which can depend on $\left(T_{w}, C_{w}, f, S\right)$. It belongs to a chosen class of functions with tuning parameters underlying for determination via the solution of the corresponding inverse problem.

Conservation of silt in suspension is governed by

$$
\begin{gathered}
\partial_{t}(\theta f)+\nabla q_{w}+\partial_{t} S_{2}=0 \\
\partial_{t} S_{2}=\Psi_{2}\left(S_{2}\right) \theta f,
\end{gathered}
$$

where $\Psi_{2}$ is the filtration function, $S_{2}$ is the deposited silt and $f$ is the silt fraction in suspension.

\subsection{Heat Energy Transport Model}

Conservation of the water heat energy is expressed in partial differential equation

$$
c_{v} \partial_{t}\left(\theta T_{w}\right)-\operatorname{div}\left(-c_{v} \overrightarrow{q_{0}} T_{w}+\theta \mathrm{D} \nabla T_{w}\right)=\sigma \theta\left(T_{m}-T_{w}\right)
$$

where $\sigma$ is a transmission coefficient, $c_{v} \vec{q} T_{w}$ being the convective part, and the diffusive part is modelled by dispersion matrix D.

\subsection{Heat Conduction in Porous Media Matrix}

A simple heat conduction model in the matrix is considered in the form 


$$
c_{m} \partial_{t} T_{m}-\lambda \Delta T_{m}=\sigma \theta\left(T_{w}-T_{m}\right),
$$

where $\lambda$ - heat conduction coefficient and $c_{m}$ - heat capacity of the matrix. In the solution of inverse problems we consider radial symmetric cylindrical sample, and thus we rewrite the considered model in cylindrical coordinates.

\section{Mathematical Model in Cylindrical Coordinates}

Our sample is with radius $\mathrm{R}$ and height $\mathrm{Z}$. We transform the mathematical model using cylindrical coordinates (r; $\mathrm{z}$ ), which reduces the difficulty of the mathematical model to a two-dimensional case due to radially symmetric boundary and initial conditions.

\subsection{Flow of Contaminated Water}

The governing partial differential equation for infiltration (in gravitational mode) reads as follows

$$
\partial_{t} \theta(h)=\frac{1}{r} \partial_{r}\left(r K\left(T_{w}, C_{w}, f, S, h\right) \partial_{r} h\right)+\partial_{z}\left(K\left(T_{w}, C_{w}, f, S, h\right)\left(\partial_{z} h-\xi\right)\right)+\partial_{t}\left(S_{1}+S_{2}\right),
$$

where

$$
\xi=1-f+f \frac{\rho_{s}}{\rho_{w}}
$$

The suspension flux in cylindrical coordinates is of the form

$$
\begin{gathered}
\overrightarrow{q_{0}}=-\left(q_{0}^{r}, q_{0}^{z}\right)^{T}, \\
q_{0}^{r}=K\left(T_{w}, C_{w}, f, S, h\right) \partial_{r} h, q_{0}^{z}=K\left(T_{w}, C_{w}, f, S, h\right)\left(\partial_{z} h-\xi\right) .
\end{gathered}
$$

\subsection{Heat Energy Transport by Water}

A matrix $D$ is of the form

$$
\mathrm{D}=\left(\begin{array}{ll}
D_{1,1} & D_{1,2} \\
D_{2,1} & D_{2,2}
\end{array}\right)=\left(\begin{array}{cc}
\alpha_{L}\left(q_{0}^{r}\right)^{2}+\alpha_{T}\left(q_{0}^{z}\right)^{2} & \left(\alpha_{L}-\alpha_{T}\right)\left(q_{0}^{r} q_{0}^{z}\right) \\
\left(\alpha_{L}-\alpha_{T}\right)\left(q_{0}^{r} q_{0}^{z}\right) & \alpha_{L}\left(q_{0}^{z}\right)^{2}+\alpha_{T}\left(q_{0}^{r}\right)^{2}
\end{array}\right) \frac{1}{\left|\overrightarrow{q_{0}}\right|}
$$

Denote by

$$
\begin{aligned}
Q T^{r} & =-q_{r} T_{w}+\theta\left(D_{1,1} \partial_{r} T_{w}+D_{1,2} \partial_{z} T_{w}\right)+D_{o} \theta \\
Q T^{z} & =-q_{z} T_{w}+\theta\left(D_{2,1} \partial_{r} T_{w}+D_{2,2} \partial_{z} T_{w}\right)+D_{o} \theta .
\end{aligned}
$$

Then, the heat energy transport reads as

$$
c_{v} \partial_{t}\left(\theta T_{w}\right)-\left(\frac{1}{r} \partial_{r}\left(r Q T^{r}\right)+\partial_{z} Q T^{z}\right)=\sigma \theta\left(T_{m}-T_{w}\right) .
$$




\subsection{Contaminant Transport}

We define contaminant fluxes $Q C^{r}, Q C^{z}$ in the same way as $Q T^{r}, Q T^{z}$, where we replace $T_{w}$ by $C_{w}$. Then we rewrite the heat transport equation replacing $T_{w}, Q T^{r}, Q T^{z}$ by $C_{w}, Q C^{r}, Q C^{z}$ and obtain contaminant transport equation in cylindrical coordinates.

\subsection{Silt Transport}

Similarly we obtain the silt fluxes $Q f^{r}, Q f^{z}$ as $Q T^{r}, Q T^{z}$, where we replace $T_{w}$ by $\mathrm{f}$. Then we obtain the silt transport equation cylindrical coordinates replacing $T_{w}, Q T^{r}, Q T^{z}$ by $f, Q f^{r}, Q f^{z}$.

\subsection{Heat Conduction In The Porous Media Matrix}

The mathematical model is

$$
\left.c_{m} \partial_{t} T_{m}-\lambda\left(\frac{1}{r} \partial_{r}\left(r \partial_{r} T_{m}\right)+\partial_{z}\left(\partial_{z} T_{m}\right)\right)\right)=\sigma \theta\left(T_{w}-T_{m}\right) .
$$

These governing equations are completed by corresponding boundary conditions including the external driven forces. For simplicity, we assume that on the boundary (or its parts) there are prescribed fluxes or values of the unknown $h, C_{w}, f, T_{w}, T_{m}$ and a combination of them. In fact, also water and heat energy transmission from external driven forces into facade could be considered and the corresponding transmission coefficient could be scaled by the solution of the inverse problem.

\section{Numerical Method}

We consider the half of cylinder crossection because of radial symmetry. The time derivation we approximate by backwards difference and then we integrate our system over the angular control volume $V_{i, j}$ with the corners $r_{i \pm 1 / 2}, z_{j \pm 1 / 2}$ and with the length $(\Delta r, \Delta z)$ of the edges.Then, our approximation linked with the inner grid point $\left(r_{i}, z_{j}\right)$ at the time $t=t_{k}$ using abbreviation $K(U):=K\left(T_{w}^{k-1}, C_{w}^{k-1}, f^{k-1}, S^{k-1}, h\right)$ is

$$
\begin{gathered}
\frac{\theta(h)-\theta\left(h^{k-1}\right)}{\tau} \Delta r \Delta z-\Delta z \frac{r_{i+\frac{1}{2}}}{r_{i}}\left[\frac{K\left(U_{i+1}\right)+K(U)}{2}\left(\frac{h_{i+1}-h}{\Delta r}\right)\right]+\Delta z \frac{r_{i-\frac{1}{2}}}{r_{i}}\left[\frac{K(U)+K\left(U_{i-1}\right)}{2}\left(\frac{h-h_{i-1}}{\Delta r}\right)\right] \\
-\Delta r\left[\frac{K\left(U_{j+1}\right)+K(U)}{2}\left(\frac{h_{j+1}-h}{\Delta z}-\xi\right)\right]+\Delta r\left[\frac{K(U)+K\left(U_{j-1}\right)}{2}\left(\frac{h-h_{j-1}}{\Delta z}-\xi\right)\right] \\
+\left(\frac{S_{1}^{j}-S_{1}^{j-1}}{\tau}+\frac{S_{2}^{j}-S_{2}^{j-1}}{\tau}\right) \Delta r \Delta \mathrm{z}=0,
\end{gathered}
$$

where only changes of $\{i, j\}$ are indicated.

\subsection{Quasi-Newton Linearization}

In each $\left(r_{i}, z_{j}\right)$ we linearize $\theta$ in terms of $h$ iteratively (with iteration parameter l) following [3] in the following way

$$
\frac{\theta\left(h^{k, l+1}\right)-\theta\left(h^{k-1}\right)}{\tau}=R^{k, l} \frac{h^{k, l+1}-h^{k, l}}{\tau}+\frac{\theta^{k, l}-\theta^{k-1}}{\tau},
$$

where

$$
R^{k, l}=\frac{\partial \theta^{k, l}}{\partial h^{k, l}}=\left(\theta_{s}-\theta_{r}\right)(1-n) \alpha\left(\alpha h^{k, l}\right)^{n-1}\left(1+\left(\alpha h^{k, l}\right)^{n}\right)^{-(m+1)}
$$

for $h^{k, l}<0$, else $R^{k, l}=0$. We stop iterations for $l=l^{*}$, when $\left|h^{k, l^{*}+1}-h^{k, l^{*}}\right| \leq$ tollerance and then we put $h^{k}$ $:=h^{k, l^{*}+1}$. Finally we replace the nonlinear term $K\left(U^{k}\right)$ by $K\left(U^{k, l}\right)$, then our approximation scheme becomes linear in 
terms of $h^{k, l+1}$. Generally, we speed the iteration by a special construction of starting point $h^{k, 0} \approx h^{k-1}$ and using suitable damping parameter in solving corresponding linearized system. Applying operator splitting method in successive solution for temperature, adsorption and deposition at the time section $t=t_{k}$ we start from $t=t_{k-1}$ and we use the obtained flow characteristics from $t=t_{k}$ for $\theta^{k}, h^{k}$ and $\vec{q}^{k}$ and for matrix $\bar{D}^{k}$. We use the same approximation strategy for the transport of contaminant, silt, and heat energy.

To obtain approximation linked with the boundary points we apply the same strategy of finite volume method, where the control volume $V_{i, j}$ is only half or quarter of the $\Delta r \Delta z$ corresponding to the inner grid points. All iterations we realize in the flow part of the model thanks to the operator splitting strategy. The other model variables are taken from the time section $k-1$. The approximation of other model equation is very similar and approximation for flux $\vec{q}_{0}$ and matrix $\vec{D}$ must be done carefully.

\section{Inverse Problems}

We choose the optimal experimental scenarios for determination of all model parameters which we restore successively. The determination of parameters $K_{S}, n, \alpha, \alpha_{L}$ and $\alpha_{T}$ we have discussed in our previous contributions (see [5]). We shortly discuss the determination of transmission coefficient $\sigma$ and matrix heat conduction $\lambda$, where we discuss the influence of $T_{w}, C_{w}, f, S$ which was neglected (because of a reduced model) in our previous contributions.

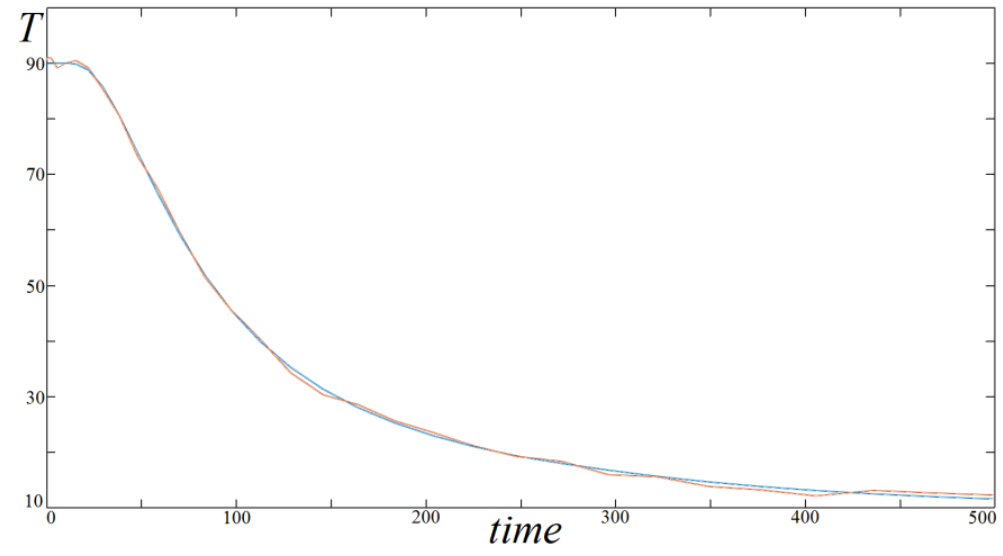

Fig. 2: Time evolution of the temperature in the center of the sample top (blue) and with random noise (red).

By means of our $3 D$ sample we can choose a suitable input/output boundary conditions to create not invasive and relative simple measurements for determination of required model parameters via the solution of inverse problems. To validate the reliability of the used scenario we compute the corresponding direct problem (when model parameters are given) and we create the data (original) corresponding to chosen characteristics. Then, we add some noise (generated by random function) to the original data and these will represent our measurements of corresponding characteristics. Finally, we forget the original model data and iteratively we construct the new (optimal) model data, minimizing the distance of computed characteristics with the original ones. We test the reliability of the obtained model parameters by choosing different starting parameters in the iteration procedure and changing the level of added noise. These facts and the sensitivity of characteristics on model parameters create the ground for suitability of suggested experimental scenario.

The determination of model parameters $\sigma, \lambda$ is based on following characteristics. We measure the temperature in the center of the sample's top. In the figure 2 we can see the time evolution of temperature decrease (blue line) with its random noise up to $1^{\circ} \mathrm{C}$ (red line). At this point (on the whole sample axis) the temperature of water and matrix are almost equal because of the following experimental scenario. The initial temperature of low saturation $(\mathrm{h}=-200)$ of the sample is $90^{\circ} \mathrm{C}$ and we infiltrate water with temperature $10^{\circ} \mathrm{C}$ through its mantel. The top together with the bottom is flow and temperature 
isolated. The obtained determination results for $\sigma, \lambda$ based on the characteristic introduced before, with different starting points of model parameters are collected in the table 1 . As we can see, the random noise up to $1^{\circ} \mathrm{C}$ causes defect up to $6 \%$. Similarly we determine the filtration and adsorption function, where the required tuning parameters we determine from measured inflow/outflow characteristics. The filtration function is considered in the form $\Psi_{2}\left(S_{2}\right)=1 /\left(a+b S_{2}\right)$ where tuning parameters $\mathrm{a}, \mathrm{b}$ have to be determined.

Table 1: Starting points and different optimal values of $\sigma, \lambda$ for different random noises up to $1^{\circ} \mathrm{C}$.

\begin{tabular}{|c|c|c|c|c|c|c|c|c|}
\hline$\sigma_{\text {start }}$ & 2 & 2 & 0.5 & 0.5 & 2 & 2 & 0.5 & 0.5 \\
\hline$\lambda_{\text {start }}$ & 0.5 & 0.5 & 0.5 & 0.5 & 0.05 & 0.05 & 0.05 & 0.05 \\
\hline$\sigma_{\text {optimal }}$ & 0.9498 & 0.9684 & 1.0515 & 1.0529 & 1.0396 & 1.0581 & 0.9407 & 1.0575 \\
\hline$\lambda_{\text {optimal }}$ & 0.09954 & 0.10089 & 0.10119 & 0.09949 & 0.10210 & 0.09933 & 0.09897 & 0.09972 \\
\hline
\end{tabular}

\subsection{Direct Solution Of Our Complex Model}

Finally, we present the solution of the system with suspension flow, temperature fields, silt deposition, and also with contaminant adsorption. In our numerical experiments, we assume the following model data ([CGS])

$\theta_{0}=0.38, \theta_{r}=0, K_{s}=2.4 \times 10^{-4}, \alpha=0.0189, n=2.81, H(0)=5, g=981, \lambda_{v}=0.03, \lambda=0.1, D_{0}=0.01$, $\alpha_{L}=1, \alpha_{T}=\frac{1}{10}, c_{v}=c_{m}=1, \rho_{m}=1, \sigma=1, \kappa=0.05, \Psi_{1}\left(C_{w}\right)=\frac{1}{1+C_{w}}, a=150$ and $b=300$.

In figure 3, figure 4 and figure 5 we present the evolution of pressure head, effective saturation, suspension temperature, matrix temperature, silt fraction in suspension, deposited silt, contaminant concentration in suspension and adsorbed contaminant in 3 time sections. We use $t_{1}=97 \mathrm{~s}, t_{2}=377 \mathrm{~s}, t_{3}=829 \mathrm{~s}$. The used parameters in the filtration function cause quick filling of the pores by the deposited silt near the infiltration boundary. In this case the continuation of the infiltration process stopped after short time. Inside the sample, there is a redistribution of the saturation leaving part of the sample suspension free as you can see in figure 5 .
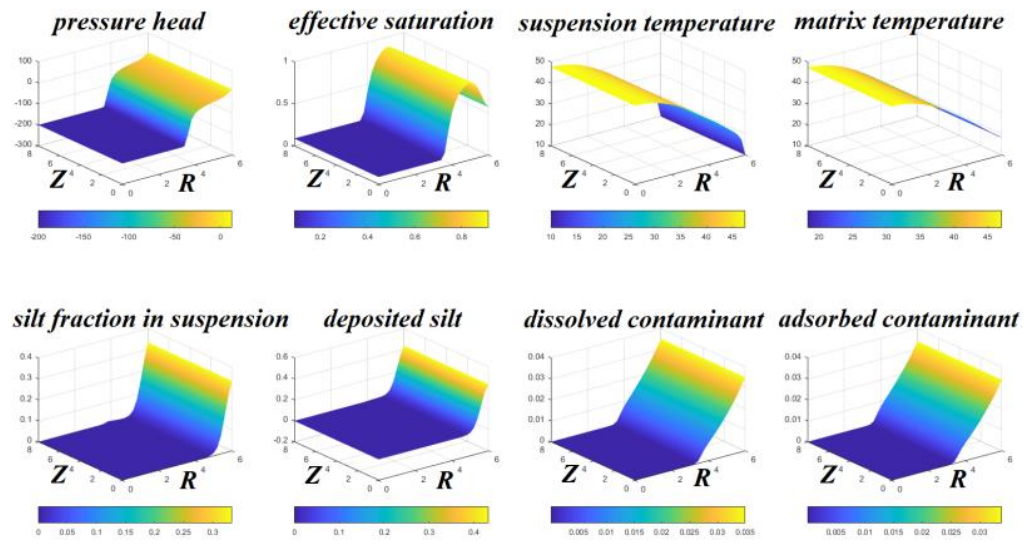

Fig. 3: Distribution of the entity values inside the sample at the time $t=97 \mathrm{~s}$. 

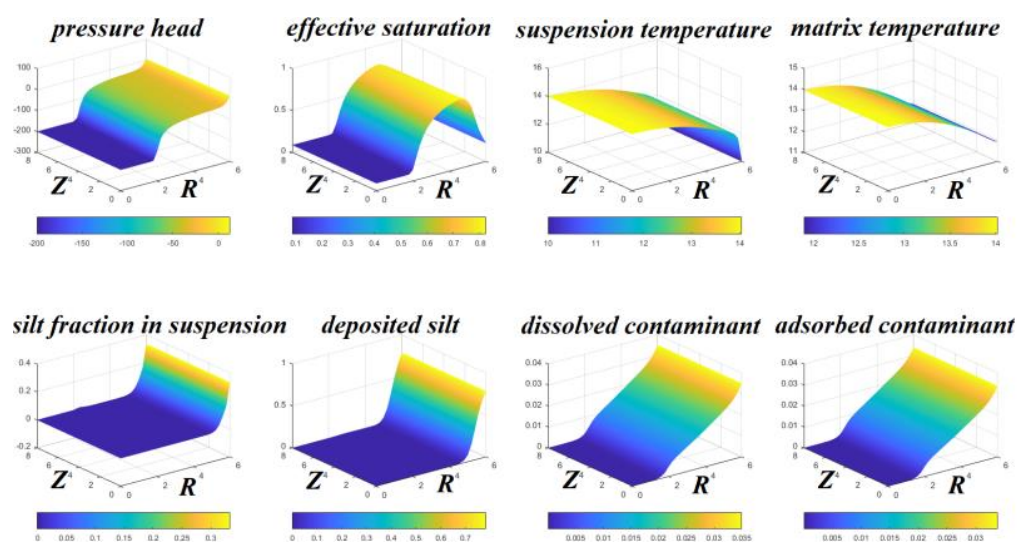

Fig. 4: Distribution of the entity values inside the sample at the time $t=377 \mathrm{~s}$.
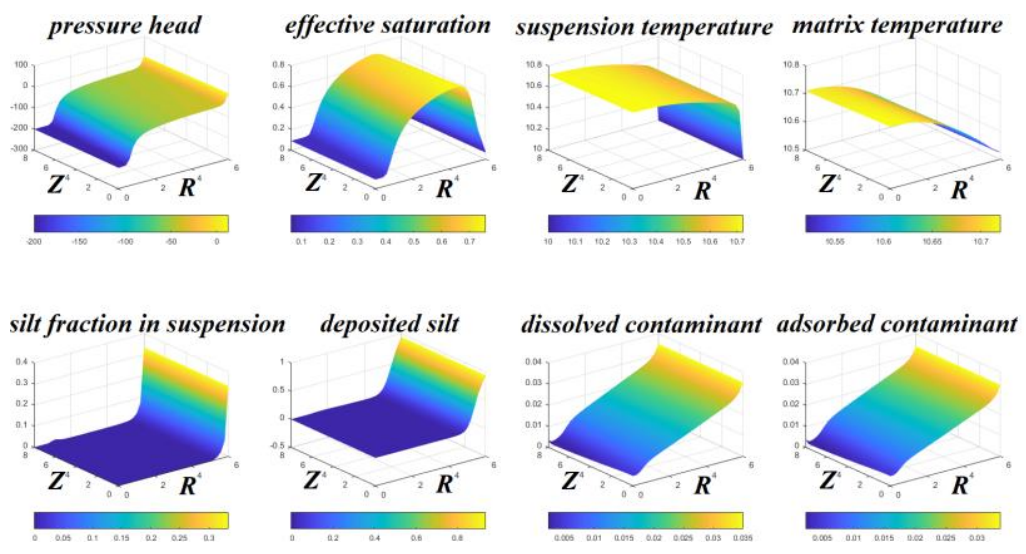

Fig. 5: Distribution of the entity values inside the sample at the time $t=829 \mathrm{~s}$.

Moreover we include figures with level contours of $h, \theta_{e f}, f$ and $S_{2}$ in the corresponding time sections as we can see in figure 6 . For time $t=97 \mathrm{~s}$ we use a red dashed line, for time $t=377 \mathrm{~s}$ we use a blue dash-dotted line and for time $t=$ $829 s$ we use a green solid line.
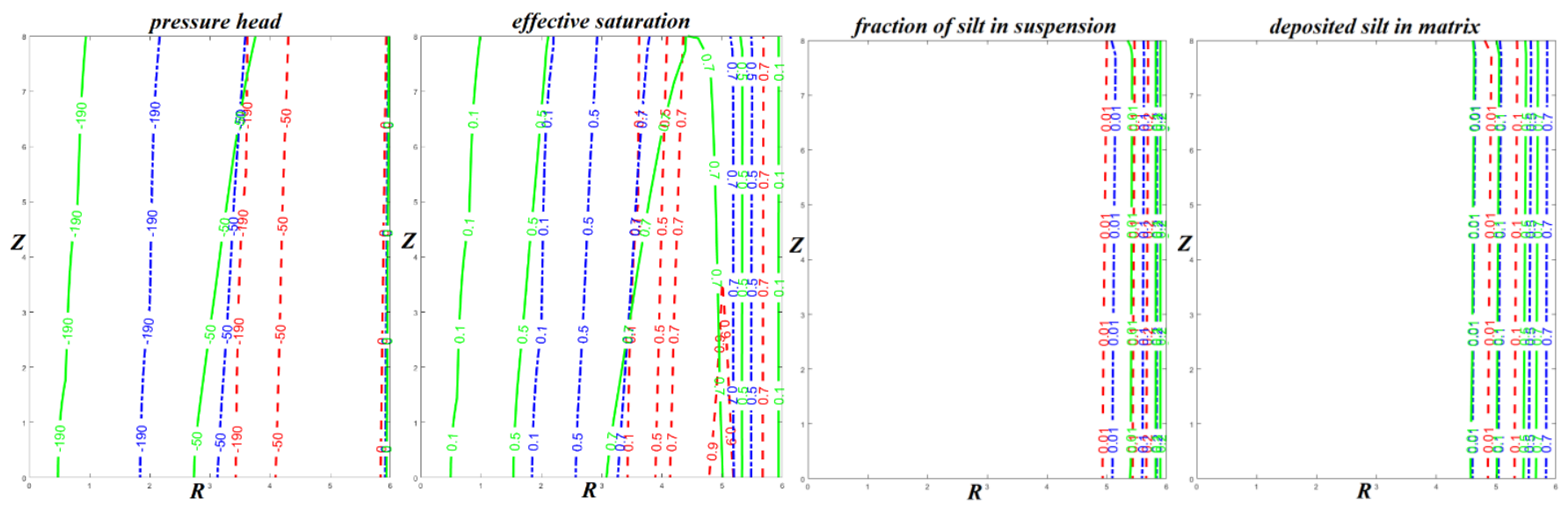

Fig. 6: Time evolution of the contours. 


\section{Conclusion}

Numerical modeling of the suspension flow and the heat and mass transport into unsaturated porous media is The mathematical model includes heat, silt, and contaminant transport with heat exchange and adsorption. The adsorbed contaminant, deposited silt, and the temperature influence the hydraulic permeability and it causes porosity degradation. efficient numerical method is developed on the base of operator splitting, flexible time discretization, and finite volume method.

A laboratory experiment scenario is proposed to determine the heat transmission coefficient and heat conductivity in the porous media matrix. In the direct solution of our complex model we demonstrated the correctness and efficiency of our numerical method for solving the direct and inverse problem.

\section{Acknowledgements}

The authors confirm financial support by the Slovak Research and Development Agency under the contracts APVV-15-0681.

\section{References}

[1] J. Bear, A. H.-D. Cheng, "Modeling Groundwater Flow and Contaminant Transport," Springer, vol. $23,2010$.

[2] J. Šimunek, M. Šejna, H. Saito, M. Sakai, M. Th. van Genuchten, "The Hydrus-1D Software Package for Simulating the Movement of Water," Heat, and Multiple Solutes in Variably Saturated Media, 2013.

[3] M. A. Celia, Z. Bouloutas, "A general mass-conservative numerical solution for the unsaturated flow equation," Water Resour., Res. 26, p. 1483-1496, 1990.

[4] M.T. Van Genuchten, "A closed form equation for predicting the hydraulic conductivity of unsaturated soils," Soil science society of Amarican Journal, vol. 44, pp. 892-898, 1980.

[5] J. Kačur, P. Mihala, M. Tóth, "Determination of soil parameters in hydraulic flow model for porous media," International journal of mechanics, vol. 11, pp. 36-42, 2017.

[6] J. Kačur, J. Minár, "A benchmark solution for infiltration and adsorption of polluted water into unsaturated-saturated porous media," Transport in porous media, vol. 97, pp. 223-239, 2013.

[7] T. L. Bergman, A. S. Lavine, F. P. Incropera, D. P. Dewitt, Fundamentals of heat and mass transfer. John Wiley and Sons, 7th edition, 2011.

[8] M. Koniorczyk, D. Gawin, "Heat and moisture transport in porous building materials containing salt," Journal of building physics, vol. 31, no. 4, pp. 279-300, 2008.

[9] N.-Z. Sun, Inverse problems in Groundwater modelling, Kluwer, Academics, Dordrecht, 1994.

[10] N.-Z. Sun, Mathematical model of groundwater pollution, Springer-Verlag, New York, 1966.

[11] A. C. Alvarez, G. Hime, J. D. Silva and D. Marchesin, Analytic regularization of an inverse filtration problem in porous media, Inverse Problems 29, 025006 (20p), 2013.

[12] Z. Mesticou, M. Kacem and PH. Dubujet, Influence of ionic strength and flow rate on silt particle deposition and release in saturated porous medium, Experiment and Modeling, Transp. Porous Med., 103, 1-24, 2014.

[13] J. P. Herzig, D. M. Leclerc and P. L. Golf, Flow of Suspensions through Porous Media - Application to Deep Filtration, Indust. Eng. Chem. 65, 8-35, 1970.

[14] P. Bedrikovetsky, A. Zeinijahromi, F.D. Siqueira, C. A. Furtado, A. L. S. de Souza, Particle Detachment Under Velocity Alternation During Suspension Transport in Porous Media, Transport Porous Media 91, 173-197, 2012.

[15] J. Kačur, P. Mihala, M. Tóth, Numerical modeling of heat exchange and unsaturated-saturated flow in porous media, Computers and Mathematics with Applications, Vol. 77, No. 9, 1668-1680, 2019. 